\title{
Optomechanical test of the Schrödinger-Newton equation
}

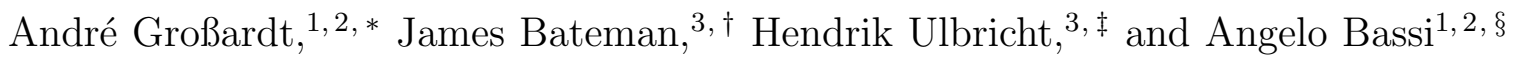 \\ ${ }^{1}$ Department of Physics, University of Trieste, 34151 Miramare-Trieste, Italy \\ ${ }^{2}$ Istituto Nazionale di Fisica Nucleare, \\ Sezione di Trieste, Via Valerio 2, 34127 Trieste, Italy \\ ${ }^{3}$ School of Physics and Astronomy, \\ University of Southampton, SO17 1BJ, United Kingdom
}

(Dated: October 7, 2015)

\begin{abstract}
The Schrödinger-Newton equation has been proposed as an experimentally testable alternative to quantum gravity, accessible at low energies. It contains self-gravitational terms, which slightly modify the quantum dynamics. Here we show that it distorts the spectrum of a harmonic system. Based on this effect, we propose an optomechanical experiment with a trapped microdisc to test the Schrödinger-Newton equation, and we show that it can be realized with existing technology.
\end{abstract}

\footnotetext{
* andre.grossardt@ts.infn.it

$\dagger \overline{\text { jbateman@soton.ac.uk }}$

$\ddagger \overline{\text { h.ulbricht@soton.ac.uk }}$

$\S$ bassi@ts.infn.it
} 
What is the gravitational field of a quantum system in a spatial superposition state? The seemingly most obvious approach, the perturbative quantization of the gravitational field in analogy to electromagnetism, makes it alluring to reply that the space-time of such a state must also be in a superposition. The non-renormalizability of said theory, however, has also inspired the hypothesis that a quantization of the gravitational field might not be necessary after all [1-3]. Rosenfeld already expressed the thought that the question whether or not the gravitational field must be quantized can only be answered by experiment: "There is no denying that, considering the universality of the quantum of action, it is very tempting to regard any classical theory as a limiting case to some quantal theory. In the absence of empirical evidence, however, this temptation should be resisted. The case for quantizing gravitation, in particular, far from being straightforward, appears very dubious on closer examination." [1]

Adopting this point of view, an alternative approach to couple quantum matter to a classical space-time is provided by a fundamentally semi-classical theory [4]; that is by replacing the source term in Einstein's field equations for the curvature of classical spacetime, energy-momentum, by the expectation value of the corresponding quantum operator [1, 5]:

$$
R_{\mu \nu}+\frac{1}{2} g_{\mu \nu} R=\frac{8 \pi G}{c^{4}}\left\langle\Psi\left|\hat{T}_{\mu \nu}\right| \Psi\right\rangle
$$

Of course, such presumption is not without complications. For instance, in conjunction with a no-collapse interpretation of quantum mechanics it would be in blatant contradiction to everyday experience [6]. Moreover, the nonlinearity that the backreaction of quantum matter with classical space-time unavoidably induces cannot straightforwardly be reconciled with quantum nonlocality in a causality preserving manner [7, 8]. Be that as it may, there is no consensus about the conclusiveness of these arguments [2, 9, 10]. The enduring quest for a theory uniting the principles of quantum mechanics and general relativity gives desirability to having access to hypotheses which could be put to an experimental test in the near future.

In the nonrelativistic limit, the assumption of fundamentally semi-classical gravity yields a nonlinear, nonlocal modification of the Schrödinger equation, commonly referred to as the Schrödinger-Newton equation [11-13]. After a suitable approximation [12], for the center of 
mass of a complex quantum system of mass $M$ in an external potential $V_{\text {ext }}$ it reads:

$$
\begin{aligned}
\mathrm{i} \hbar \frac{\partial}{\partial t} \psi(t, \mathbf{r}) & =\left(\frac{\hbar^{2}}{2 M} \nabla^{2}+V_{\text {ext }}+V_{g}[\psi]\right) \psi(t, \mathbf{r}) \\
V_{g}[\psi](t, \mathbf{r}) & =-G \int \mathrm{d}^{3} r^{\prime}\left|\psi\left(t, \mathbf{r}^{\prime}\right)\right|^{2} I_{\rho_{c}}\left(\mathbf{r}-\mathbf{r}^{\prime}\right)
\end{aligned}
$$

The self-gravitational potential $V_{g}$ depends on the wavefunction, and hence renders the equation nonlinear. The function $I_{\rho_{c}}$, which models the mass distribution of the considered system, will be defined below.

The Schrödinger-Newton equation has primarily been discussed in the context of gravitationally induced quantum state reduction [14, 15]. Its relevance for a possible experimental test of the necessity to quantize the gravitational field was pointed out by Carlip [3]. First ideas how to test such kind of nonlinear, self-gravitational effects focused on the spreading of a free wavefunction in matter-wave interferometry experiments [3, 16 21]. Recently, an experimental test has been proposed by Yang et al. [22], based on the internal dynamics of a squeezed coherent ground state of a micron-sized silicon particle in a harmonic potential.

Here we propose a novel experiment, studying the spectrum of such a harmonically trapped microparticle. The advantage of our proposal is that it needs neither squeezing nor state tomography. Therefore, despite the fact that effects are of the same order of magnitude than those studied in Ref. [22], the prospects of its practical realization in the soon future are significantly better.

We show that for a suitable choice of mass and frequencies the energy levels become state dependent, due to the self-gravity term $V_{g}[\psi]$ in Eq. (2), thus resulting in a non-degenerate line spacing of the energy levels of the harmonic oscillator. This is schematically depicted in Fig. 1.

\section{THEORY}

In matter-wave interferometry, even large molecules can approximately be considered as single, pointlike particles, meaning that the wavefunction is wide in comparison to the extent of the considered quantum system. In this case, the function $I_{\rho_{c}}$ in Eq. (2) reduces to the Coulomb-like potential $M^{2} /\left|\mathbf{r}-\mathbf{r}^{\prime}\right|$. This changes if the wavefunction for the center of mass

cannot be treated as wide, as it is the usual situation in optomechanical experiments. In that instance, one must take the mass distribution $\rho_{c}$ of the constituents relative to the 


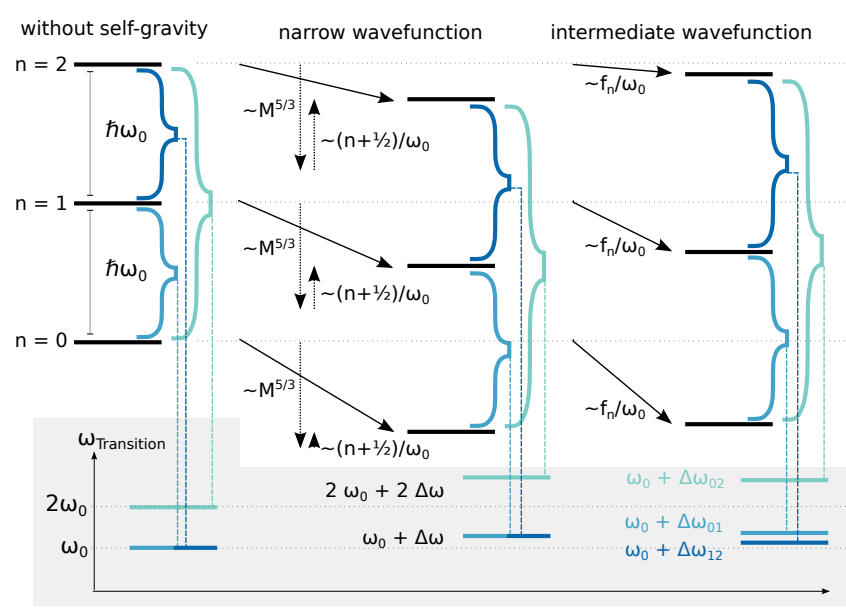

FIG. 1. Schematic overview of the effect of the Schrödinger-Newton equation on the spectrum. The top part shows the first three energy eigenvalues and their shift due to the first order perturbative expansion of the Schrödinger-Newton potential. The bottom part shows the resulting spectrum of transition frequencies. In the narrow wavefunction regime (middle part), all energy levels are shifted down by an $n$-independent value minus an $n$-proportional contribution that scales with the inverse trap frequency. In the intermediate regime, where the wavefunction width becomes comparable to the localization length scale of the nuclei, this $n$-proportionality does no longer hold, leading to a removal of the degeneracy in the spectrum.

center of mass into account. The general shape of $I_{\rho_{c}}$ is

$$
I_{\rho_{c}}(\mathbf{d})=\int \mathrm{d}^{3} u \mathrm{~d}^{3} v \frac{\rho_{c}(\mathbf{u}) \rho_{c}(\mathbf{v}-\mathbf{d})}{|\mathbf{u}-\mathbf{v}|} .
$$

It has been pointed out by Yang et al. 22] that, for a sufficiently narrow wavefunction, the crystalline structure of matter becomes significant. Provided that the atomic mass density can be modeled by a Gaussian distribution, and the microparticle as a whole has a spherical structure of radius $R$ much larger than the extent of the wavefunction, we get approximately [23]

$$
I_{\rho_{c}}(d) \approx \frac{6 M^{2}}{5 R}+\frac{M m}{d} \operatorname{erf}\left(\frac{d}{\sqrt{2} \sigma}\right) .
$$

Here, and in the following, we denote by $m$ the atomic mass, and by $M$ the mass of the whole microparticle. The localization of the nuclei, $\sigma$, is related to the Debye-Waller B-factor by $\sigma=2 \pi \sqrt{B}$. Values for $B$ at different temperatures can be found in Refs. [24, 25] for most elemental crystals. 
The external potential is now supposed to be a harmonic trap with frequency $\omega_{0}$ in $x$ direction, and we assume the wavefunction to separate in the three spatial dimensions [26]. The additional self-gravitational potential leads to a shift of the energy levels from their unperturbed values at $E_{n}^{(0)}=\hbar \omega_{0}(1 / 2+n)$. If the system is in a stationary state of the trap, and the mass is such that the self-gravitational potential is weak, as is the case in any realistic experimental situation, the energy shift is well approximated by a first-order perturbative expansion in the gravitational constant $G$ :

$$
\Delta E_{n}=\left\langle\psi_{n}^{(0)}\left|V_{g}\left[\psi_{n}^{(0)}\right](\mathbf{r})\right| \psi_{n}^{(0)}\right\rangle+\mathcal{O}\left(G^{2}\right)
$$

Strictly speaking, this is a twofold approximation, first by taking the unperturbed state $\psi_{n}^{(0)}$ as the source of the gravitational potential, which renders $V_{g}$ a linear potential, and then applying ordinary perturbation theory. Inserting the energy eigenstates of the harmonic oscillator, and introducing the dimensionless parameter $\alpha=2 \sigma \sqrt{M \omega_{0} / \hbar}$, the energy shift can be written

$$
\Delta E_{n}=-\frac{G \hbar m}{4 \sigma^{3} \omega_{0}} f_{n}(\alpha),
$$

with the state, mass, and frequency dependent functions $f_{n}$ yet to be determined.

We first consider the situation of Ref. [22], where the potential $V_{g}$ was simplified further by taking the limit of a narrow wavefunction, and Taylor expanding $I_{\rho_{c}}$ to quadratic order in $\left|\mathbf{r}-\mathbf{r}^{\prime}\right|$. In this case, the function $f_{n}$ takes the form

$$
f_{n}^{\text {narrow }}(\alpha)=\propto M^{5 / 3} \omega_{0}-\frac{4}{3} \sqrt{\frac{2}{\pi}}\left(n+\frac{1}{2}\right) .
$$

Obviously, the transition frequency $\omega_{0}$ between adjacent energy levels will not be affected by the $n$-independent part, while the $n$-proportional term leads to a shift

$$
\Delta \omega_{\mathrm{SN}}=\sqrt{\frac{2}{\pi}} \frac{G m}{3 \omega_{0} \sigma^{3}} .
$$

This frequency shift is, however, independent of $n$, therefore leaving the degeneracy of the spectrum intact, according to which all energy transitions with the same $\Delta n$ correspond to the same spectral line at $\Delta n\left(\omega_{0}+\Delta \omega_{\mathrm{SN}}\right)$.

The experimental situation of Ref. [22], where the frequency is high enough to allow for the wavefunction to be approximated as narrow, hides the true behavior of the energy levels. This becomes evident if we consider the scenario where the width of the wavefunction 
is comparable to the localization $\sigma$ of the nuclei, and hence the quadratic approximation becomes inaccurate. The wavefunction width is characterized by the factor $\alpha$ defined above, which relates the width of the ground state to $\sigma$. Small values, $\alpha \ll 1$, correspond to wide wavefunctions and large values, $\alpha \gg 1$, to narrow ones.

For now, consider only the case where the trap frequency is lowered in one dimension, but the wavefunction is kept narrow in the remaining two. Then, in the intermediate regime, where $\alpha$ is of the order of unity, the function $f_{n}$ can be approximated (see Ref. [23] for a more thorough derivation)

$$
f_{n}(\alpha) \approx \text { const. }+\alpha^{3} \sqrt{\frac{2}{\pi}} \int_{0}^{\infty} \mathrm{d} \zeta \exp \left(-\frac{\alpha^{2} \zeta^{2}}{2}\right) P_{n}(\alpha \zeta)\left(\frac{\operatorname{erf}(\sqrt{2} \zeta)}{2 \zeta}-\sqrt{\frac{2}{\pi}}\right)
$$

where "const." refers to $n$-independent terms, and the polynomials $P_{n}$ are defined by

$$
P_{n}(z)=\frac{\mathrm{e}^{-z^{2} / 2}}{\sqrt{2 \pi}\left(2^{n} n !\right)^{2}} \int_{-\infty}^{\infty} \mathrm{d} \xi \mathrm{e}^{-2 \xi^{2}} H_{n}(\xi)^{2}\left(\mathrm{e}^{2 z \xi} H_{n}(\xi-z)^{2}+\mathrm{e}^{-2 z \xi} H_{n}(\xi+z)^{2}\right),
$$

with the Hermite polynomials $H_{n}$. The integrals in $f_{n}$ can be evaluated analytically for low $n$ [27]. One ends up with the frequency shift

$$
\begin{aligned}
\Delta \omega_{n_{1} n_{2}}^{\text {interm. }} & =\Delta \omega_{\mathrm{SN}} g_{n_{1} n_{2}}(\alpha) \\
g_{n_{1} n_{2}}(\alpha) & =\frac{3}{8} \sqrt{2 \pi}\left(f_{n_{1}}(\alpha)-f_{n_{2}}(\alpha)\right),
\end{aligned}
$$

which now depends not only on the difference $\Delta n$ but explicitly on $n_{1}$ and $n_{2}$. This is the effect we are interested in, which can be observed experimentally. $\Delta \omega_{\text {SN }}$ contains the material properties, while $g_{n_{1} n_{2}}(\alpha)$ depends only on the total mass $M$ and trap frequency $\omega_{0}$.

The functions $g_{n_{1} n_{2}}$ are plotted in Fig. 2 for $\Delta n=1$ and $n_{1}$ ranging from 0 to 12 . As one can see in the plot, while they tend to zero for small $\alpha$ (wide wavefunctions) and to $\Delta n$ for large $\alpha$ (narrow wavefunctions), for values $1 \lesssim \alpha \lesssim 10$ there is a significant dependence on the actual state, leading to a substantial deviation from the degenerate structure of the spectrum.

A fully three-dimensional analysis, giving up the approximation of a wavefunction that is narrow in two dimensions, can only be obtained numerically. For an axially symmetric wavefunction that is in the ground state for the transverse directions the details can be found in App. A of Ref. [23]. There it has been shown that the effect stays the same in quality and order of magnitude also in this three-dimensional situation. 


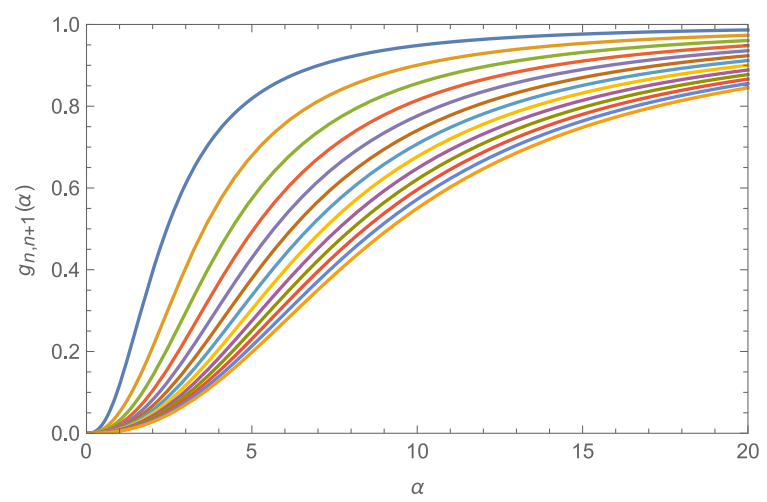

FIG. 2. Plot of the coefficient function $g_{n_{1} n_{2}}(\alpha)$ for the lowest 13 transitions between harmonic oscillator eigenstates with $\Delta n=1$. $n$ increases from top to bottom, the blue curve belonging to $n_{1}=0$. The parameter $\alpha$ characterizes the wavefunction width. Small values of $\alpha$ (wide wavefunctions) correspond to smaller masses (for a given frequency) and therefore weaker selfgravity. In the limit of narrow wavefunctions (large $\alpha$ ) the lines become degenerate again, all $g_{n_{1} n_{2}}$ tending to $\Delta n=1$.

\section{PROPOSAL FOR EXPERIMENT}

We propose to measure this effect by interrogating optomechanically the motion of a single micron-sized superconducting osmium mirror in a dilution refrigeration-cooled linear Paul ion trap, as shown schematically in Fig. 3. The Schrödinger-Newton effect will be probed with the longitudinal motion ( $x$-direction in Fig. 3) of the trapped osmium microdisc.

While best known for atomic and molecular ions, the first demonstration of Paul traps included a $20 \mu \mathrm{m}$ particle [28], and a recently renewed interest has shown single micron-scale particles at $100 \mathrm{~Hz}$ frequencies with single-charge resolution [29, 30]. Longitudinal frequency can be orders of magnitude lower than the transverse [31, 32], which accurately embodies our theoretical description that the wave function remains narrow in two dimensions.

\section{A. Magnitude of the expected effect}

Requiring that the ground-state wavefunction be in the intermediate regime, $\alpha \approx 5$, yields $M=\hbar /(2 \sigma / \alpha)^{2} / \omega_{0} \approx 10^{16} \mathrm{u} /\left(\omega_{0} / 2 \pi \mathrm{s}^{-1}\right)$. Values of $\Delta \omega_{\mathrm{SN}}$ for selected materials can be found in Tab. I. We choose osmium, which has superconducting critical temperature $T_{\mathrm{C}}=700 \mathrm{mK}$, favorable Debye-Waller B-factor [25], and offers the smallest particle for a 


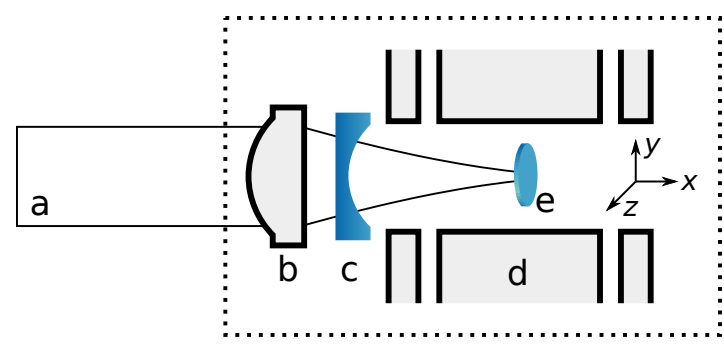

FIG. 3. Schematic of proposed experiment showing a) optical access, b) mode-matching lens, c) concave cavity mirror, d) ion trap electrode structure, and e) electrically levitated superconducting disc. The disc acts as a concave cavity mirror and forms an optomechanical cavity with mirror (c) to read the position of the disc. The apparatus is enclosed in a dilution refrigerator to reduce thermal noises. The longitudinal direction, which will be used to investigate the self-gravity modification of the mechanical harmonic oscillator energy levels, is along the $x$-axis.

\begin{tabular}{|c|c|c|c|c|}
\hline Material & $m / \mathrm{u}$ & $\rho / \mathrm{g} / \mathrm{cm}^{3}$ & $\sigma / \mathrm{pm}$ & $\Delta \omega_{\mathrm{SN}} / \mathrm{s}^{-1}$ \\
\hline Silicon & $28.086^{\mathrm{a}}$ & $2.329^{\mathrm{a}}$ & $6.96^{\mathrm{b}}$ & $0.00246 /\left(\omega_{0} / \mathrm{s}^{-1}\right)$ \\
\hline Tungsten & $183.84^{\mathrm{a}}$ & $19.30^{\mathrm{a}}$ & $3.48^{\mathrm{b}}$ & $0.128 /\left(\omega_{0} / \mathrm{s}^{-1}\right)$ \\
\hline Osmium & $190.23^{\mathrm{a}}$ & $22.57^{\mathrm{a}}$ & $2.77^{\mathrm{c}}$ & $0.264 /\left(\omega_{0} / \mathrm{s}^{-1}\right)$ \\
\hline Gold & $196.97^{\mathrm{a}}$ & $19.32^{\mathrm{a}}$ & $4.66^{\mathrm{b}}$ & $0.0574 /\left(\omega_{0} / \mathrm{s}^{-1}\right)$ \\
\hline
\end{tabular}

a Reference [33], ${ }^{\mathrm{b}}$ Reference [24, ${ }^{\mathrm{c}}$ Reference [25]

TABLE I. Relevant material properties for selected elements. $\sigma=2 \pi \sqrt{B}$ is defined as in the text and depends on the Debye-Waller B-factor. Values are at $T=100 \mathrm{mK}$. We give $\Delta \omega_{\mathrm{SN}}$, which determines the magnitude of the effect according to Eq. 10$)$, depending on the trap frequency $\omega_{0}$.

given mass. The spectral lines are plotted for different mass osmium particles at a trap frequency $\omega_{0}=2 \pi \times 10 \mathrm{~s}^{-1}$ in Fig. 4. The split between adjacent spectral lines scales with $1 / \omega_{0}$, just like the mass for which the effect is most pronounced. For $M=10^{15} \mathrm{u}$, corresponding to an osmium particle (density $\rho=22.57 \mathrm{~g} / \mathrm{cm}^{3}$ ) of diameter $5.2 \mu \mathrm{m}$, we predict a frequency splitting $\Delta f \sim 0.1 \mathrm{mHz}$.

A spherical particle would have radius comparable with typical laser wavelengths, making it impractical for use in a concave-convex cavity. Instead, the superconductor should be a thin disk $\gtrsim 3 \mu \mathrm{m}$ in diameter and $\sim 1 \mu \mathrm{m}$ thick [34]. Finesse in cavities with wavelength-scale mirrors is limited by mirror size and orientation stability [35]. 


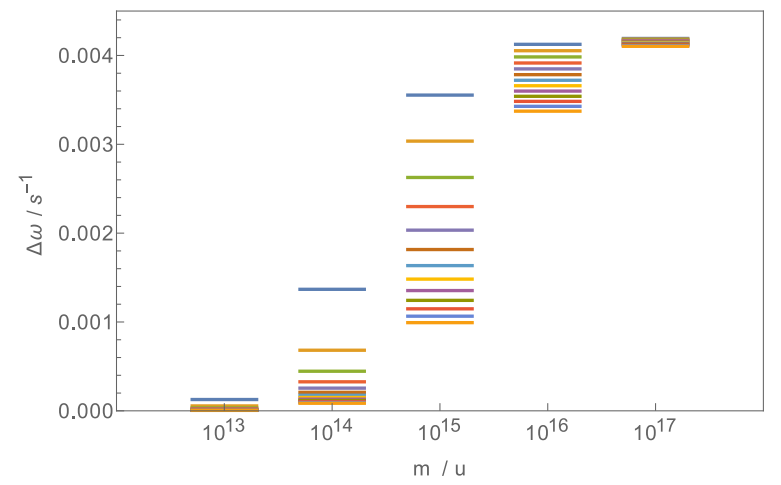

FIG. 4. The resulting frequency spectrum for osmium at $T=100 \mathrm{mK}$ at trap frequency $\omega_{0}=$ $2 \pi \times 10 \mathrm{~s}^{-1}$ with $\Delta n=1$. At low masses, self-gravity becomes negligible. At high masses all spectral lines are degenerate, shifted by $\Delta \omega_{\mathrm{SN}}$. The intermediate regime, where a significant splitting appears, spans about 3 orders of magnitude in mass.

\section{B. Competing effects}

All competing heating effects must produce a damping rate which is low compared with the frequency shift we expect: sub-Hertz heating rates have been demonstrated in roomtemperature conventional traps [36] and cryogenics reduces heating rates substantially, as demonstrated in microfabricated traps [37]; rates depend strongly on microfabrication processes, and improvements to the levels we require are likely. Indeed, already a cryogenicallycooled conventional trap should fulfill our requirements with existing technology.

Decoherence routes include interaction with blackbody radiation: however, for radiation frequencies below the superconductor gap energy there is no absorption [38], so by ensuring environmental temperature much less than superconductor critical temperature, interaction with ambient photons is exclusively via Rayleigh scattering. To reach this regime requires dilution refrigerator temperatures and we assume $T=100 \mathrm{mK}$. Using the particle as a mirror, not a sub-wavelength particle, means there is negligible Rayleigh scattering of laser light [39], which would otherwise be a decoherence mechanism. Rayleigh scattering rate decreases sharply for long wavelengths, and we find negligible probability of even a single scattering event [40]. Decoherence from collisions with background gas is also negligible for the low pressures $\left(P \lesssim 10^{-10}\right.$ mbar) in the UHV cryo-pumped environment [41]. 


\section{Optomechanical readout}

Lack of a long-lived internal state in our particle, such as those employed in sideband resolved manipulation of trapped atomic ions, means that many techniques for engineering Fock states in ion traps [42] cannot be applied here. Instead we appeal to optomechanics: inspired by cantilever experiments [43], where a microfabricated cantilever provides one mirror of a high-finesse cavity, we use the levitated metallic superconducting particle of sufficient reflectivity as one mirror in a tightly focused, plano-concave, high optical Q cavity. Cantilever position can be monitored at the shot-noise limit [44, and protocols have been proposed to prepare [45] and reconstruct [46] Fock states in these systems.

There are experiments with levitated hybrid nanoparticle ion-optomechanical systems, albeit with sub-wavelength particles [47]. Creation of Fock states of mechanical motion or phonon number states for $10^{14} \mathrm{u}$ particles has previously been proposed for a magneticallylevitated superconducting microsphere coupled to a quantum circuit [41], while its experimental demonstration is yet to be shown.

\section{CONCLUSION}

We predict a new self-gravity effect to shift the energy states of a massive mechanical oscillator. We propose an experimental scenario which makes use of the best parts of the mature technology of levitation of ions in Paul traps, the cavity enhanced optical position readout and the use of superconducting materials to avoid competing heating effects for instance by black body radiation. Our proposal is based on technology available today. However, the different techniques have to be combined. It does not require the preparation and tomography of squeezed states of the mechanical harmonic oscillator. Mechanical Fock states need to be prepared and read out, which is technology under intense development as of today and has yet to be achieved. The self-gravity induced energy level splitting effect survives also for states above the mechanical vacuum state, and hence particularly for thermal states, which means cooling to the ground state is not required as such. Therefore our proposed experiment is feasible with existing technology. 


\section{ACKNOWLEDGMENTS}

The authors gratefully acknowledge funding and support from the John Templeton foundation (grant 39530). AG acknowledges funding from the German Research Foundation (DFG). JB and HU acknowledge support from the UK funding body EPSRC (EP/J014664/1). HU acknowledges financial support from the Foundational Questions Institute (FQXi). AB acknowledges financial support from the EU project NANOQUESTFIT, INFN, and the University of Trieste (grant FRA 2013).

[1] L. Rosenfeld, Nucl. Phys. 40, 353 (1963),

[2] J. Mattingly, in Einstein Studies Volume 11. The Universe of General Relativity, Einstein Studies, edited by A. J. Kox and J. Eisenstaedt (Birkhäuser, Boston, 2005) Chap. 17, pp. $327-338$.

[3] S. Carlip, Class. Quantum Grav. 25, 154010 (2008), arXiv:0803.3456 [gr-qc],

[4] It needs to be stressed, once again, that in this approach the coupling between gravity and quantum matter is fundamentally semi-classical. This should not be confused with models where Eq. (1) is considered an approximation to some quantum theory of gravity [48].

[5] C. Møller, in Colloques Internationaux CNRS, Vol. 91, edited by A. Lichnerowicz and M.-A. Tonnelat (CNRS, Paris, 1962).

[6] D. N. Page and C. D. Geilker, Phys. Rev. Lett. 47, 979 (1981).

[7] K. Eppley and E. Hannah, Found. Phys. 7, 51 (1977).

[8] N. Gisin, Helv. Phys. Acta 62, 363 (1989).

[9] C. Kiefer, Quantum Gravity, 2nd ed., International Series of Monographs on Physics, Vol. 124 (Clarendon Press, Oxford, 2007).

[10] M. Albers, C. Kiefer, and M. Reginatto, Phys. Rev. D 78, 064051 (2008), arXiv:0802.1978 $[\mathrm{gr}-\mathrm{qc}]$.

[11] D. Giulini and A. Großardt, Class. Quantum Grav. 29, 215010 (2012), arXiv:1206.4250 [gr-qc],

[12] D. Giulini and A. Großardt, New J. Phys. 16, 075005 (2014), arXiv:1404.0624 [gr-qc],

[13] M. Bahrami, A. Großardt, S. Donadi, and A. Bassi, New J. Phys. 16, 115007 (2014), arXiv:1407.4370 [quant-ph]. 
[14] L. Diósi, Phys. Lett. A 105, 199 (1984).

[15] R. Penrose, Phil. Trans. R. Soc. A 356, 1927 (1998),

[16] D. Giulini and A. Großardt, Class. Quantum Grav. 28, 195026 (2011), arXiv:1105.1921 [gr-qc].

[17] J. R. van Meter, Class. Quantum Grav. 28, 215013 (2011), arXiv:1105.1579 [quant-ph],

[18] D. Giulini and A. Großardt, Class. Quantum Grav. 30, 155018 (2013), arXiv:1212.5146 [gr-qc],

[19] S. Colin, T. Durt, and R. Willox, "Crucial tests of macrorealist and semi-classical gravity models with freely falling mesoscopic nanospheres," (2014), arXiv:1402.5653 [quant-ph].

[20] M. Arndt and K. Hornberger, Nat. Phys. 10, 271 (2014).

[21] S. Eibenberger, S. Gerlich, M. Arndt, M. Mayor, and J. Tüxen, Phys. Chem. Chem. Phys. 15, 14696 (2013).

[22] H. Yang, H. Miao, D.-S. Lee, B. Helou, and Y. Chen, Phys. Rev. Lett. 110, 170401 (2013), arXiv:1210.0457 [gr-qc],

[23] A. Großardt, J. Bateman, H. Ulbricht, and A. Bassi, "Effects of Newtonian gravitational selfinteraction in harmonically trapped quantum systems," (2015), arXiv:1510.01262 [quant-ph].

[24] V. F. Sears and S. A. Shelley, Acta Crystallogr. Sect. A 47, 441 (1991).

[25] H. X. Gao and L.-M. Peng, Acta Crystallogr. Sect. A 55, 926 (1999).

[26] Note that according to the full Eq. (2) even an initially separable state will evolve into a nonseparable one. Only with the approximation (5) the self-gravitational potential becomes separable.

[27] We have been using Mathematica to obtain analytical results for the functions $f_{n}$ up to $n=14$. The computation time increases super-proportionally with $n$, making it infeasible to obtain results for higher $n$.

[28] R. F. Wuerker, H. Shelton, and R. V. Langmuir, J. Appl. Phys. 30, 342 (1959).

[29] D. Gerlich, Hyperfine Interactions 146-147, 293 (2003).

[30] S. Schlemmer, S. Wellert, F. Windisch, M. Grimm, S. Barth, and D. Gerlich, Appl. Phys. A 78, 629 (2004).

[31] M. Drewsen and A. Brøner, Phys. Rev. A 62, 045401 (2000).

[32] H. Landa, M. Drewsen, B. Reznik, and A. Retzker, New J. Phys. 14, 093023 (2012).

[33] K. A. Olive et al. (Particle Data Group), Chin. Phys. C 38, 090001 (2014), http://pdg.lbl. gov/2014/AtomicNuclearProperties. 
[34] While we considered a spherical particle in the theory section, the shape of the particle contributes only to the $n$-independent shift of the energy levels. For the effect we are interested in, only the mass distribution of the nuclei inside the particle is relevant.

[35] D. Kleckner, W. T. Irvine, S. S. Oemrawsingh, and D. Bouwmeester, Phys. Rev. A 81, 043814 (2010).

[36] G. Poulsen, Y. Miroshnychenko, and M. Drewsen, Phys. Rev. A 86, 051402 (2012).

[37] J. Labaziewicz, Y. Ge, P. Antohi, D. Leibrandt, K. R. Brown, and I. L. Chuang, Phys. Rev. Lett. 100, 013001 (2008).

[38] M. Dressel, Advances in Condensed Matter Physics 2013 (2013), article ID 104379.

[39] G. Guccione, M. Hosseini, S. Adlong, M. Johnsson, J. Hope, B. Buchler, and P. Lam, Phys. Rev. Lett. 111, 183001 (2013).

[40] See supplemental material.

[41] O. Romero-Isart, L. Clemente, C. Navau, A. Sanchez, and J. Cirac, Phys. Rev. Lett. 109, 147205 (2012).

[42] D. Leibfried, R. Blatt, C. Monroe, and D. Wineland, Rev. Mod. Phys. 75, 281 (2003).

[43] M. Aspelmeyer, T. J. Kippenberg, and F. Marquardt, Rev. Mod. Phys. 86, 1391 (2014).

[44] T. P. Purdy, R. W. Peterson, and C. A. Regal, Science 339, 801 (2013).

[45] C. Galland, N. Sangouard, N. Piro, N. Gisin, and T. J. Kippenberg, Phys. Rev. Lett. 112, $143602(2014)$.

[46] M. R. Vanner, I. Pikovski, and M. Kim, Ann. Phys. (Berlin) 527, 15 (2015).

[47] J. Millen, P. Z. G. Fonseca, T. Mavrogordatos, T. S. Monteiro, and P. F. Barker, Phys. Rev. Lett. 114, 123602 (2015), arXiv:1407.3595 [physics.optics].

[48] C. Anastopoulos and B. L. Hu, New J. Phys. 16, 085007 (2014), arXiv:1403.4921 [quant-ph]. 


\section{Supplemental Material for "Gravitational fine structure of harmonically trapped particles according to the Schrödinger-Newton equation"}

\section{RAYLEIGH SCATTERING OF BLACKBODY RADIATION BY SUPERCON- DUCTING MICRODISC}

For radiation frequencies below the superconductor gap energy [S1] there is no absorption; by ensuring the radiation temperature is much less than the gap energy, we can assume that no photons with energy exceeding the gap are encountered. Under these conditions, the polarizability of the sub-wavelength particle is $\chi=3 V$ where $V$ is the volume of the particle. The Rayleigh scattering cross-section is $\sigma_{R}=k^{4} \chi^{2} / 6 \pi$ where $k=\omega / c$ is the wavenumber. Integrating over the blackbody energy density $u(T, \omega)$ we find the total rate of photon scattering

$$
\Gamma_{R}=\int_{0}^{\infty} \mathrm{d} \omega \frac{\sigma_{R} c u(T, \omega)}{\hbar \omega}=30720 \pi^{5} \zeta(7) \times c \frac{\chi^{2}}{\lambda_{\mathrm{T}}^{7}} \approx 10^{7} \times c \frac{\chi^{2}}{\lambda_{\mathrm{T}}^{7}}
$$

where $\zeta$ is the Riemann zeta function $(\zeta(7) \approx 1.01)$ and $\lambda_{\mathrm{T}}=h c / k_{B} T$ is the typical wavelength of the thermal radiation at temperature $T$. For $T=100 \mathrm{mK}$, we find $\lambda_{\mathrm{T}} \approx 14 \mathrm{~cm}$.

Therefore, for both a sphere of $V=\frac{4}{3} \pi(1 \mu \mathrm{m})^{3}$ and a disc of $V=\frac{\pi}{4}(1 \mu \mathrm{m})(3 \mu \mathrm{m})^{2}$ the scattering rate is $\Gamma_{R} \sim 10^{-12} \mathrm{~s}^{-1}$.

\section{REFERENCES TO SUPPLEMENTAL MATERIAL}

[S1] M. Dressel, Advances in Condensed Matter Physics 2013 (2013), article ID 104379. 